\title{
Outcomes of Inhaled Amikacin and Clofazimine-Containing Regimens for Treatment of Refractory Mycobacterium avium Complex Pulmonary Disease
}

\author{
Bo-Guen Kim ${ }^{1}{ }^{(D}$, Hojoong Kim ${ }^{1}$ (D) O. Jung Kwon ${ }^{1}$, Hee Jae Huh ${ }^{2}$, Nam Yong Lee ${ }^{2}$, \\ Sun-Young Baek ${ }^{3}$, Insuk Sohn ${ }^{3}$ and Byung Woo Jhun ${ }^{1, *}$ \\ 1 Division of Pulmonary and Critical Care Medicine, Department of Medicine, Samsung Medical Center, \\ Sungkyunkwan University School of Medicine, Seoul 06351, Korea; kbg1q2w3e@gmail.com (B.-G.K.); \\ hjk3425@skku.edu (H.K.); ojkwon@skku.edu (O.J.K.) \\ 2 Department of Laboratory Medicine and Genetics, Samsung Medical Center, Sungkyunkwan University \\ School of Medicine, Seoul 06351, Korea; pmhhj77@gmail.com (H.J.H.); micro.lee@samsung.com (N.Y.L.) \\ 3 Statistics and Data Center, Research Institute for Future Medicine, Samsung Medical Center, \\ Seoul 06351, Korea; sun0.baek@gmail.com (S.-Y.B.); insuks@gmail.com (I.S.) \\ * Correspondence: byungwoo.jhun@gmail.com; Tel.: +82-02-3410-3429; Fax: +82-02-3410-3849
}

Received: 20 August 2020; Accepted: 11 September 2020; Published: 14 September 2020

\begin{abstract}
Limited data are available regarding optimal treatment for refractory Mycobacterium avium complex-pulmonary disease (MAC-PD). We evaluated outcomes of inhaled amikacin (AMK) with clofazimine (CFZ) regimens as an add-on salvage therapy for refractory MAC-PD. We retrospectively analyzed 52 patients with refractory MAC-PD, characterized by persistently positive sputum cultures despite $>6$ months of treatment. Thirty-five $(67 \%)$ patients had M. intracellulare-PD, and $17(33 \%)$ patients had M. avium-PD. Twenty-seven (52\%) patients received the salvage therapy for $\geq 12$ months, whereas $25(48 \%)$ patients were treated for $<12$ months due to adverse effects or other reasons. Seventeen $(33 \%)$ patients had culture conversion: $10(10 / 27)$ in the $\geq 12$-month treatment group and seven $(7 / 25)$ in the $<12$-month treatment group $(p=0.488)$. Microbiological cure, defined as maintenance of culture negativity, was achieved in 12 (23\%) patients; six (6/12) with accompanying symptomatic improvement were considered to have reached cure. Clinical cure, defined as symptomatic improvement with $<3$ consecutive negative cultures, was achieved in three $(6 \%)$ patients. Overall, 15 (29\%) patients achieved favorable outcomes, including microbiological cure, cure, and clinical cure. Inhaled AMK with CFZ may provide favorable outcomes in some patients with refractory MAC-PD. However, given the adverse effects, more effective strategies are needed to maintain these therapeutic regimens.
\end{abstract}

Keywords: Mycobacterium avium; refractory; inhalation; amikacin; clofazimine

\section{Introduction}

Nontuberculous mycobacteria (NTM) are ubiquitous organisms that cause chronic disease, and the burdens of NTM-pulmonary disease (PD) are increasing worldwide [1-3]. A guideline of the American Thoracic Society/Infectious Disease Society of America recommended multidrug therapy for Mycobacterium avium complex (MAC)-PD consisting of a macrolide (i.e., clarithromycin or azithromycin), ethambutol, and rifamycin (i.e., rifampin or rifabutin) with or without addition of an injectable aminoglycoside $[4,5]$. However, treatment outcomes are unsatisfactory, and a substantial proportion of patients remain culture-positive with refractory MAC-PD [6-9]. A recent meta-analysis 
showed that the overall treatment success rate is as low as 60\% [7] and that multidrug regimens are poorly tolerated. Therefore, improved therapeutic options are needed for refractory MAC-PD.

The recent guidelines of the British Thoracic Society and Cystic Fibrosis Foundation/Society recommend regimens that include inhaled amikacin (AMK) or other oral agents [10,11]. In addition, data have recently been reported regarding inhaled AMK with clofazimine (CFZ) for the treatment of refractory NTM-PD [12,13]. Notably, the CONVERT trial showed the benefit of adding liposomal AMK inhalation for refractory MAC-PD [14]. In addition, synergistic effects of inhaled AMK and CFZ against NTM species have been observed in vitro [15]. Nevertheless, limited data are available regarding the efficacy of regimens that include inhaled AMK and CFZ in refractory MAC-PD. Therefore, we evaluated the treatment outcomes of inhaled AMK and CFZ regimens as salvage therapy for refractory MAC-PD.

\section{Materials and Methods}

\subsection{Study Patients}

We retrospectively analyzed 52 patients with refractory MAC-PD, defined as having persistently positive cultures after $>6$ months of standardized treatment, who received a regimen containing inhaled AMK with CFZ from January 2016 to June 2018 at the Samsung Medical Center (clinicaltrials.gov identifier NCT00970801) (Figure 1). All patients met the diagnostic criteria for MAC-PD [4]. Data from five of these 52 patients were published previously in another report [12].

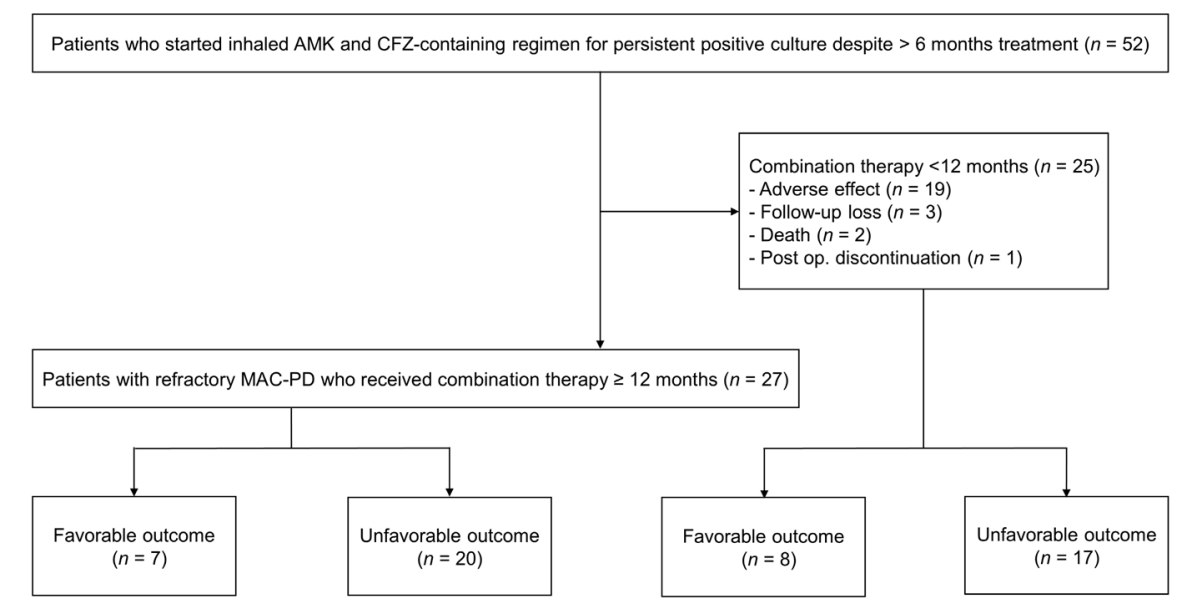

Figure 1. Flow diagram of study population.

This study was approved by the Institutional Review Board (approval no. 2019-07-121). Data were collected with informed consent from patients included in the prospective study, and data on the patients included in this study were analyzed retrospectively. Follow-up data were last updated on 10 July 2019.

\subsection{Inhaled $A M K$ and $C F Z$ Therapy}

Commercially available intravenous AMK sulfate solution $(250 \mathrm{mg} / \mathrm{mL})$ was used for inhalation, via a model NE-C28 compressor nebulizer (Omron Colin Co., Ltd., Tokyo, Japan). Before September 2016, patients were treated with $500 \mathrm{mg}$ of inhaled AMK once daily. However, several ototoxicity events occurred, and thus the protocol was changed from $500 \mathrm{mg}$ once daily to $500 \mathrm{mg}$ three times weekly [12]. Oral CFZ was initially prescribed at $100 \mathrm{mg}$ once daily.

Patients underwent laboratory and audiometry examinations at approximately 3-month intervals at an outpatient clinic. Nephrotoxicity was defined as an increase in serum creatinine of $\geq 0.5 \mathrm{mg} / \mathrm{dL}$ from baseline [16]. Ototoxicity was defined as hearing loss of $\geq 10 \mathrm{~dB}$ at two or more consecutive 
frequencies, hearing loss of $\geq 20 \mathrm{~dB}$ at one isolated frequency, or loss of a response at any three consecutive frequencies on a posttreatment audiogram, in either ear at any frequency [16].

\subsection{Assessment of Symptomatic, Radiological, and Microbiological Response}

Symptomatic responses were assessed by the chronic obstructive pulmonary disease assessment test (CAT) score as improved, unchanged, or worsened, based on a change in CAT score of $\geq 2$ points from baseline [17]. A chest radiograph or computed tomography (CT) image was performed at the initiation of therapy, as well as during follow-up. The radiological form was categorized as fibrocavitary (FC) or nodular bronchiectatic (NB) [18]. According to the presence of a cavitary lesion, NB was additionally classified as a cavitary or non-cavitary form.

Sputum acid-fast bacillus (AFB) smears and cultures were performed using standard methods at 1-3 months intervals [19]. Specimens were cultured both on 3\% Ogawa solid medium (Shinyang, Seoul, Korea) and in liquid broth medium in mycobacterial growth indicator tubes (MGIT; Becton, Dickinson and Co., Sparks, MD, USA). For semi-quantitative culture analysis, each culture was scored using methods described in previous studies [20,21]. NTM species identification was conducted via nested multiplex polymerase chain reaction and a reverse-hybridization assay of the internal transcribed spacer region (AdvanSureTM Mycobacteria GenoBlot Assay; LG Life Sciences, Seoul, Korea). Drug susceptibility testing was performed using the broth microdilution method. MAC isolates were evaluated in terms of their resistance to clarithromycin (minimum inhibitory concentration (MIC $\geq 32 \mu \mathrm{g} / \mathrm{mL}$ ) and to AMK (MIC $\geq 64 \mu \mathrm{g} / \mathrm{mL}$ ) [22].

\subsection{Evaluation of Treatment Outcomes}

Treatment outcomes were assessed at the end of the inhaled AMK and CFZ therapy as follows [23]. "Culture conversion" was defined as at least three consecutive negative sputum cultures after treatment, collected at least four weeks apart. The time of conversion was defined as the date of the first negative culture. "Microbiological cure" was defined as no positive cultures of causative species after culture conversion until the time of outcome measurement. "Clinical cure" was defined as improved symptoms during treatment without available culture to prove culture conversion or microbiological cure ( $<3$ consecutive negative cultures). "Cure" was defined as fulfillment of both clinical and microbiological cure. In this study, microbiological cure, cure, and clinical cure were classified as favorable outcomes. For analysis, we categorized the patients according to the treatment duration of 12 months based on recommended minimum treatment period [5].

\subsection{Statistical Analysis}

Data were presented as numbers (percentages) or medians (interquartile ranges (IQR)). Data were compared by the Mann-Whitney U test for continuous variables and the chi-squared test or Fisher's exact test for categorical variables. We analyzed the trend of serial changes of semi-quantitative sputum culture positivity by generalized estimating equations. Logistic regression analysis with backward stepwise selection (with $p<0.05$ for entry and $p>0.10$ for removal of variables) was used to identify independent factors associated with a favorable outcome. Differences were considered statistically significant at $p<0.05$. All statistical analyses were performed using SPSS (IBM SPSS Statistics ver. 25, Chicago, IL, USA).

\section{Results}

\subsection{Baseline Characteristics}

Among the 52 patients, the median age was 59 (IQR, 51-70) years, and $48 \%$ of them were women (Table 1). More than half (56\%) of the patients were never-smokers. The most common underlying condition was previous, treated, pulmonary tuberculosis $(52 \%)$, followed by chronic pulmonary aspergillosis (33\%). Approximately two-thirds (67\%) of the patients had M. intracellulare-PD, 
while one-third (33\%) had M. avium-PD. Twenty-seven patients (52\%) had the NB form of MAC-PD with (10/27) or without (17/27) a cavity, while the remaining had the FC form. Positive sputum AFB smears were identified in $34(65 \%)$ patients. Macrolide resistance was identified in 25 (48\%) patients, although none had pre-treatment isolates resistant to AMK (Table 1).

Table 1. Characteristics of the 52 patients at initiation of inhaled AMK with CFZ regimens.

\begin{tabular}{|c|c|}
\hline Characteristics & Value \\
\hline Age, years & $59(51-70)$ \\
\hline Female & $25(48 \%)$ \\
\hline Body mass index, $\mathrm{kg} / \mathrm{m}^{2}$ & $20.7(18.3-22.2)$ \\
\hline Weight, $\mathrm{kg}$ & $54(48-62)$ \\
\hline Never-smoker & $29(56 \%)$ \\
\hline \multicolumn{2}{|l|}{ Underlying condition } \\
\hline Previous pulmonary tuberculosis & $27(52 \%)$ \\
\hline Chronic pulmonary aspergillosis & $17(33 \%)$ \\
\hline Chronic obstructive pulmonary disease & $14(27 \%)$ \\
\hline Previous lung cancer $*$ & $3(6 \%)$ \\
\hline Diabetes mellitus & $4(8 \%)$ \\
\hline Chronic liver disease & $2(4 \%)$ \\
\hline Chronic kidney disease & $1(2 \%)$ \\
\hline Rheumatoid disease & $1(2 \%)$ \\
\hline Other malignancy ${ }^{+}$ & $2(4 \%)$ \\
\hline \multicolumn{2}{|l|}{ Etiologic organism } \\
\hline M. intracellulare & $35(67 \%)$ \\
\hline M. avium & $17(33 \%)$ \\
\hline \multicolumn{2}{|l|}{ Radiologic findings } \\
\hline Nodular bronchiectatic form & $27(52 \%)$ \\
\hline With cavity & $10 / 27(37 \%)$ \\
\hline Without cavity & $17 / 27(63 \%)$ \\
\hline Fibrocavitary form & $25(48 \%)$ \\
\hline \multicolumn{2}{|l|}{ Laboratory findings } \\
\hline Sputum AFB smear positivity & $34(65 \%)$ \\
\hline Serum albumin, g/dL & $4.1(3.8-4.4)$ \\
\hline C-reactive protein, $\mathrm{mg} / \mathrm{dL}$ & $0.6(0.1-1.7)$ \\
\hline Erythrocyte sedimentation rate, $\mathrm{mm} / \mathrm{h}$ & $30.0(18.0-67.3)$ \\
\hline $\mathrm{FEV}_{1}, \%$ & $68(53-81)$ \\
\hline FVC, \% & $74(56-90)$ \\
\hline Macrolide resistance, $\geq 32 \mu / \mathrm{mL}$ & $25(48 \%)$ \\
\hline MIC level of AMK & $16(16-32)$ \\
\hline $4-16 \mu \mathrm{g} / \mathrm{mL}$ & $37(71 \%)$ \\
\hline $32 \mu \mathrm{g} / \mathrm{mL}$ & $15(29 \%)$ \\
\hline
\end{tabular}

Data are presented as $n$ (\%) or median (interquartile range). AMK, amikacin; CFZ, clofazimine; $\mathrm{AFB}$, acid-fast bacilli; $\mathrm{FEV}_{1}$, forced expiratory volume-one second; FVC, forced vital capacity; MIC, minimum inhibitory concentration. * No patients had active cancer after lobectomy $(n=2)$ or chemoradiotherapy $(n=1) .{ }^{+}$Pancreatic cancer $(n=1)$ and thymoma $(n=1)$.

\subsection{Antibiotic Treatment Regimens}

All patients had persistent positive cultures after previous antibiotic therapy, which was received for a median treatment duration of 28.5 (IQR, 20.3-55.5) months. Before initiation of the inhaled AMK with CFZ therapy, all patients received azithromycin and ethambutol-based regimens that included rifamycin $(96 \%)$ or moxifloxacin $(25 \%)$ (Table 2). Thirty $(58 \%)$ patients had previously received injectable aminoglycoside for a median of 7.0 (IQR, 5.5-12.0) months. 
Table 2. Antibiotic treatment regimens.

\begin{tabular}{ccc}
\hline Variables & $N=\mathbf{5 2}$ & Treatment Duration (Months) \\
\hline Drugs before starting inhaled AMK and CFZ therapy & & \\
Azithromycin & $52(100 \%)$ & $27.0(19.0-56.0)$ \\
Ethambutol & $52(100 \%)$ & $27.0(16.0-56.0)$ \\
Rifamycin & $50(96 \%)$ & $28.0(19.0-56.0)$ \\
Moxifloxacin & $13(25 \%)$ & $12.0(6.0-16.0)$ \\
Aminoglycoside injection & $30(58 \%)$ & $7.0(5.5-12.0)$ \\
AMK & $2(4 \%)$ & $3.0,8.0$ \\
Streptomycin & $28(54 \%)$ & $7.0(6.0-12.0)$ \\
Total duration before starting AMK and CFZ therapy & $52(100 \%)$ & $28.5(20.3-55.5)$ \\
\hline Inhaled AMK and CFZ therapy & & \\
Total duration of inhaled AMK and CFZ & $52(100 \%)$ & $11.9(4.7-18.8)$ \\
$\geq 12$ months & $27 / 52(52 \%)$ & $18.7(12.6-26.4)$ \\
$<12$ months & $25 / 52(48 \%)$ & $4.6(1.2-6.6)$ \\
Total duration of inhaled AMK & $52(100 \%)$ & $12.9(7.2-20.6)$ \\
Total duration of CFZ & $52(100 \%)$ & $12.5(5.5-18.9)$ \\
Azithromycin & & \\
Ethambutol & $51(98 \%)$ & $15.4(12.4-20.9)$ \\
Rifamycin & $50(96 \%)$ & $16.3(12.7-22.2)$ \\
Moxifloxacin & $11(21 \%)$ & $4.0(3.0-13.0)$ \\
Linezolid & $10(19 \%)$ & $8.5(3.0-14.2)$ \\
Total duration after starting AMK and CFZ therapy & $1(2 \%)$ & 12.4 \\
\hline Companion drugs & $52(100 \%)$ & $15.4(12.5-21.2)$ \\
\hline
\end{tabular}

Data are presented as $n$ (\%) or median (interquartile range). AMK, amikacin; CFZ, clofazimine.

Regarding the dosage of inhaled AMK, 35 (67\%) patients began treatment with $500 \mathrm{mg}$ three times weekly; nine $(17 \%)$ patients changed from $500 \mathrm{mg}$ once daily to three times weekly because of a protocol change in September 2016 in our institution; and $8(16 \%)$ patients began treatment with $500 \mathrm{mg}$ once daily. All patients received $100 \mathrm{mg}$ of oral CFZ at the initiation of treatment. After initiation of the inhaled AMK and CFZ therapy, 27 (52\%) patients maintained the regimen for $\geq 12$ months, while 25 (48\%) patients discontinued the regimen in less than 12 months (Figure 1 ). The median duration of AMK and CFZ therapy in all study patients was 11.9 (IQR, 4.7-18.8) months. Companion drugs, including azithromycin (98\%), ethambutol (96\%), rifamycin (21\%), moxifloxacin $(19 \%)$, and linezolid (2\%), were used in combination with inhaled AMK and CFZ during the study period (Table 2). At the time of starting AMK and CFZ combination therapy, we usually used CFZ instead of rifamycin due to concerns about hepatotoxicity caused by concomitant use of CFZ and rifamycin. In cases with development of macrolide resistance, azithromycin was maintained for its anti-inflammatory effect in our institution.

\subsection{Treatment Response of Study Patients}

Treatment responses were evaluated at the end of the inhaled AMK and CFZ therapy (Table 3). Of the 52 patients, symptomatic and radiological improvement were observed in $25(48 \%)$ and $17(33 \%)$, respectively. Twenty-two (42\%) patients had at least one negative culture; sputum culture conversion was achieved by $17(33 \%)$ patients, although nine (53\%) of these 17 patients had clarithromycin-resistant isolates. Microbiological cure was achieved in $12(23 \%)$ patients, including five (5/12, 42\%) patients with clarithromycin-resistant isolates, but only six (6/12) of them had accompanying symptomatic improvement and were thus considered to have achieved cure. Three $(6 \%)$ patients who showed improvement of symptoms with $<3$ consecutive negative cultures were considered to exhibit clinical cure; one $(33 \%)$ of the three had clarithromycin-resistant isolates. Overall, $15(29 \%)$ patients achieved a favorable outcome, although $47 \%$ (7/15) of these patients had clarithromycin-resistant isolates. Sputum culture conversion was achieved after a median of 3.0 months of treatment, and microbiological cure 
was achieved after a median of 1.1 months of treatment (Supplementary Figure S1A,B). In addition, among the 25 patients who initially had clarithromycin-resistant isolates, culture conversion, microbiological cure, and favorable outcome were achieved in $36 \%(9 / 25), 20 \%(5 / 25)$, and $28 \%$ (7/25), respectively.

Table 3. Treatment response at the end of the inhaled AMK- and CFZ-containing regimens.

\begin{tabular}{|c|c|}
\hline Variables & $N=52$ \\
\hline \multicolumn{2}{|l|}{ Symptomatic response by CAT score change } \\
\hline Improved & $25(48 \%)$ \\
\hline Unchanged & $11(21 \%)$ \\
\hline Worsened & $16(31 \%)$ \\
\hline \multicolumn{2}{|l|}{ Radiological response } \\
\hline Improved & $17(33 \%)$ \\
\hline Unchanged & $20(39 \%)$ \\
\hline Worsened & $15(29 \%)$ \\
\hline \multicolumn{2}{|l|}{ Microbiological response } \\
\hline At least one sputum negative culture & $22(42 \%)$ \\
\hline Time to at least one culture negative, months & $5.1(1.0-10.1)$ \\
\hline Culture conversion & $17(33 \%)$ \\
\hline Time to culture conversion, months & $3.0(1.0-9.2)$ \\
\hline Microbiological cure & $12(23 \%)$ \\
\hline Cure & $6(12 \%)$ \\
\hline Clinical cure & $3(6 \%)$ \\
\hline Favorable outcome & $15(29 \%)$ \\
\hline \multicolumn{2}{|l|}{ Microbiological response in $\geq 12$ months treatment group $(n=27)$} \\
\hline Culture conversion & $10 / 27(37 \%)$ \\
\hline Time to culture conversion, months & $3.0(0.9-9.3)$ \\
\hline Microbiological cure & $7 / 27(26 \%)$ \\
\hline Cure & $2 / 27(7 \%)$ \\
\hline Clinical cure & $0 / 27(0 \%)$ \\
\hline \multicolumn{2}{|l|}{ Microbiological response in $<12$ months treatment group $(n=25)$} \\
\hline Culture conversion & $7 / 25(28 \%)$ \\
\hline Time to culture conversion, months & $3.0(1.0-9.7)$ \\
\hline Microbiological cure & $5 / 25(20 \%)$ \\
\hline Cure & $4 / 25(16 \%)$ \\
\hline Clinical cure & $3 / 25(12 \%)$ \\
\hline Death * & $6(12 \%)$ \\
\hline Time from starting inhaled AMK and CFZ to death, months & $11.1(5.8-20.5)$ \\
\hline
\end{tabular}

Data are presented as $n$ (\%) or median (interquartile range). AMK, amikacin; CFZ, clofazimine; CAT, chronic obstructive pulmonary disease assessment test. * Two patients died before completion of 12 months treatment of AMK and CFZ therapy due to progression of MAC-PD. The remaining four patients died of pneumonia $(n=2)$, pancreatic cancer $(n=1)$, and unknown cause $(n=1)$.

According to the culture results on solid medium, 46 (46/52, 88\%) patients had $\geq 1+$ AFB culture positivity at the time of initiation of therapy. Thirteen $(13 / 27,48 \%)$ patients had $\geq 1+$ AFB culture positivity after 12 months of inhaled AMK and CFZ therapy. There was a significant reduction in semiquantitative sputum culture positivity after treatment $(p<0.001)$. In addition, among the 35 patients who did not achieve culture conversion during the study period, semiquantitative culture positivity also tended to decrease significantly $(p=0.012)$ (Table S1). 
For semi-quantitative culture analysis, each culture was scored as follows: (i) negative, no growth in liquid or solid medium; (ii) liquid only, growth in liquid medium only; (iii) trace, growth of $<50$ colonies on solid medium; (iv) 1+, growth of 50 to 100 colonies on solid medium; (v) 2+, growth of 100 to 200 colonies on solid medium; (vi) 3+, growth of 200 to 500 colonies on solid medium; and (vii) $4+$, growth of $>500$ colonies on solid medium.

\subsection{Adverse Effects Associated with Inhaled AMK and CFZ}

During the study period, $24(46 \%)$ and 14 (27\%) patients experienced adverse effects associated with inhaled AMK and CFZ, respectively (Table 4). Seventeen patients discontinued inhaled AMK after a median of 3.0 (IQR, 1.1-6.6) months, and seven patients had a change in inhaled AMK dosage: $500 \mathrm{mg}$ once daily to $500 \mathrm{mg}$ three times weekly $(n=4), 500 \mathrm{mg}$ three times weekly to $500 \mathrm{mg}$ twice weekly $(n=2)$, and $500 \mathrm{mg}$ three times weekly to $250 \mathrm{mg}$ three times weekly $(n=1)$.

Table 4. Adverse effects associated with inhaled AMK and CFZ therapy in 52 study patients.

\begin{tabular}{cccc}
\hline & Discontinuation & Dose Change & Total \\
\hline Amikacin inhalation & & & \\
Ototoxicity & $12(23 \%)$ & $5(10 \%)$ & $17(33 \%)$ \\
Fatigue & $2(4 \%)$ & $0(0 \%)$ & $2(4 \%)$ \\
Tinnitus & $1(2 \%)$ & $0(0 \%)$ & $1(2 \%)$ \\
Dizziness & $1(2 \%)$ & $0(0 \%)$ & $1(2 \%)$ \\
Nausea & $1(2 \%)$ & $0(0 \%)$ & $1(2 \%)$ \\
Hoarseness & $0(0 \%)$ & $1(2 \%)$ & $1(2 \%)$ \\
Nephrotoxicity & $0(0 \%)$ & $1(2 \%)$ & $1(2 \%)$ \\
Total & $17(33 \%)$ & $7(14 \%)$ & $24(46 \%)$ \\
Clofazimine & & & \\
Skin color change & $1(2 \%)$ & $9(17 \%)$ & $10(19 \%)$ \\
Loss of appetite & $1(2 \%)$ & $0(0 \%)$ & $2(4 \%)$ \\
Diarrhea & $0(0 \%)$ & $1(2 \%)$ & $1(2 \%)$ \\
Fatigue & $1(2 \%)$ & $0(0 \%)$ & $1(2 \%)$ \\
Total & $1(2 \%)$ & $0(0 \%)$ & $1(2 \%)$ \\
Hepatotoxicity $(>3$ times the normal level) & $4(8 \%)$ & $10(19 \%)$ & $14(27 \%)$ \\
\hline
\end{tabular}

Data are presented as $n(\%)$. AMK, amikacin; CFZ, clofazimine. ${ }^{*}$ Median time from starting inhaled AMK to discontinuation was 3.0 months. ${ }^{\dagger}$ AMK dosage changes: $500 \mathrm{mg}$ once daily to $500 \mathrm{mg}$ three times weekly $(n=4)$, $500 \mathrm{mg}$ three times weekly to $500 \mathrm{mg}$ two times weekly $(n=2)$, and $500 \mathrm{mg}$ three times weekly to $250 \mathrm{mg}$ three times weekly $(n=1) .{ }^{\ddagger}$ Median time from starting CFZ to discontinuation was 1.7 months.

Four (8\%) patients discontinued CFZ after 1.7 (IQR, 1.2-5.2) months, and ten (19\%) patients had dosages of CFZ changed from $100 \mathrm{mg}$ once daily to $50 \mathrm{mg}$ once daily.

\subsection{Factors Associated with a Favorable Outcome}

Factors associated with a favorable outcome of refractory MAC-PD were explored (Table 5). In multivariable analysis, low ESR was significantly associated with a favorable outcome in all patients (adjusted odds ratio, 0.950; 95\% confidence interval, 0.914-0.988). 
Table 5. Univariate and multivariable analyses of factors associated with favorable outcome $(N=52)$.

\begin{tabular}{|c|c|c|c|c|c|}
\hline \multirow[b]{2}{*}{ Variable } & \multirow{2}{*}{$\begin{array}{c}\text { Favorable Outcome * } \\
\qquad(n=15)\end{array}$} & \multicolumn{2}{|c|}{ Univariable } & \multicolumn{2}{|c|}{ Multivariable } \\
\hline & & $\begin{array}{l}\text { Unadjusted OR } \\
(95 \% \mathrm{CI})\end{array}$ & $p$ Value & $\begin{array}{l}\text { Adjusted OR } \\
(95 \% \mathrm{CI})\end{array}$ & $p$ Value \\
\hline Age $\leq 65$ years & $2(13 \%)$ & $4.432(0.871-22.550)$ & 0.073 & & \\
\hline Female & $9(60 \%)$ & $1.969(0.581-6.673)$ & 0.277 & & \\
\hline Body mass index, $\mathrm{kg} / \mathrm{m}^{2}$ & & $1.058(0.857-1.308)$ & 0.599 & & \\
\hline Never-smoker & $10(67 \%)$ & $1.895(0.542-6.628)$ & 0.317 & & \\
\hline No previous pulmonary tuberculosis & $8(53 \%)$ & $1.345(0.404-4.477)$ & 0.630 & & \\
\hline No chronic obstructive pulmonary disease & $14(93 \%)$ & $7.583(0.894-64.331)$ & 0.063 & & \\
\hline M. avium (reference: $M$. intracellulare) & $8(53 \%)$ & $3.556(1.006-12.562)$ & 0.049 & & \\
\hline Negative sputum AFB smear & $6(40 \%)$ & $1.389(0.401-4.806)$ & 0.604 & & \\
\hline No cavity & $7(47 \%)$ & $2.362(0.679-8.222)$ & 0.177 & & \\
\hline No macrolide resistance & $8(53 \%)$ & $1.083(0.325-3.602)$ & 0.897 & & \\
\hline Amikacin MIC $<32 \mu \mathrm{g} / \mathrm{mL}$ & $11(73 \%)$ & $1.163(0.303-4.461)$ & 0.825 & & \\
\hline Treatment duration $\geq 12$ months & $10(67 \%)$ & $2.353(0.672-8.239)$ & 0.181 & & \\
\hline $\mathrm{FEV}_{1}>60 \%$ & $14(93 \%)$ & $9.545(1.132-80.506)$ & 0.038 & & \\
\hline $\mathrm{ESR}, \mathrm{mm} / \mathrm{h}^{+}$ & & $0.950(0.914-0.988)$ & 0.010 & $0.950(0.914-0.988)$ & 0.010 \\
\hline $\mathrm{CRP}, \mathrm{mg} / \mathrm{dL}^{+}$ & & $0.228(0.057-0.904)$ & 0.035 & & \\
\hline
\end{tabular}

OR, odds ratio; CI, confidence interval; $\mathrm{AFB}$, acid-fast bacilli; MIC, minimum inhibitory concentration; $\mathrm{FEV}_{1}$, forced expiratory volume-one second; ESR, erythrocyte sedimentation rate; CRP, C-reactive protein. * favorable outcome included microbiological cure, cure, and clinical cure. ${ }^{\dagger}$ ESR and CRP were measured at the time of starting combination therapy.

\section{Discussion}

In this study, more than one-quarter (29\%) of patients with refractory MAC-PD achieved a favorable outcome after initiation of inhaled AMK- and CFZ-containing regimens. Approximately one-third (33\%) of patients had culture conversion, and microbiological cure occurred in $23 \%$ of patients. Our data suggest that regimens containing inhaled AMK with CFZ may yield favorable outcomes in some patients with refractory MAC-PD.

One of the most notable findings in our study was the high proportion of patients with strains that had pretreatment drug resistance. Approximately half (48\%) of our patients had clarithromycin-resistant MAC strains at the initiation of the inhaled AMK and CFZ therapy. In previous studies of refractory MAC-PD, which showed culture conversion rates similar to our study, the proportions of patients with macrolide-resistant MAC strains were not high. In a study that included 23 patients with refractory MAC-PD who were treated with inhaled AMK therapy, 39\% of patients had clarithromycin-resistant strains prior to treatment, and the conversion rate was $43 \%$ [24]. In a recent CONVERT study that evaluated the efficacy of AMK liposome inhalation suspension for patients with refractory MAC-PD, culture conversion was achieved by $29 \%$ of patients; however, only $22.9 \%$ of patients had pretreatment clarithromycin resistance [14]. Macrolides are the cornerstone of MAC-PD therapy, and development of macrolide resistance, mainly due to point mutations in the $23 \mathrm{~S}$ rRNA gene, is associated with poor treatment outcomes [25-29]. Thus, given that 36\% (9/25) of our patients with clarithromycin-resistant strains experienced culture conversion, inhaled AMK- and CFZ-containing regimens may be worth consideration as treatment strategies, especially for patients with refractory MAC-PD.

AMK is one of the most important antibiotics for treatment of MAC-PD. Only $6.8-10.4 \%$ of clinical strains of MAC are reportedly resistant to AMK [30], and a recent study indicated that use of aminoglycosides for $\geq 3$ months was associated with treatment success in cavitary MAC-PD [31]. Inhaled AMK has been used to reduce adverse effects associated with long-term use of injectable AMK and to increase the therapeutic effect through lung absorption [32]. However, studies regarding the efficacy of inhaled AMK for refractory NTM-PD have shown varying culture conversion rates of $18-67 \%[12,24,32,33]$. These discrepancies may have been related to various epidemiological or clinical factors in the study cohorts. Notably, our patients had a long previous median treatment period of 28.5 months; $67 \%$ (35/52) of patients had cavitary lesions, which are known to be associated with poor response [18], and this high percentage of cavitary disease cases potentially contributed to the less-than-satisfactory treatment outcomes of many of our patients. Unfortunately, limited data are available regarding whether the differences in effectiveness of inhaled AMK depend on certain patient 
characteristics, such as the presence of cavitary lesions. Therefore, further studies are needed regarding the efficacy of inhaled AMK for refractory MAC-PD.

CFZ has a number of advantages for treatment of NTM-PD, including its long half-life, slow metabolic elimination, ability to achieve high concentrations in macrophages, and rapid localization within phagocytes. Laboratory studies have demonstrated synergistic effects of CFZ and AMK against NTM [15], and CFZ has been reported to be effective in treatment of MAC-PD [13,34,35]. However, unlike the laboratory findings, these clinical studies yielded inconsistent results. Moreover, appropriate criteria have not been established regarding an optimal MIC threshold of CFZ for MAC-PD, and further research is warranted on this issue.

In our study, approximately half $(48 \%)$ of the patients discontinued regimens containing inhaled AMK and CFZ within 12 months, mostly due to adverse effects. Eventually, 33\% (17/52) of patients discontinued the inhaled AMK, and 8\% (4/52) of patients discontinued CFZ. The discontinuation rate of inhaled AMK tended to be higher in our study than in previous reports [12,24]. In a previous study, $27 \%$ (21/77) of patients with refractory NTM-PD discontinued inhaled AMK [12], and only 8\% (2/26) of patients with refractory MAC-PD discontinued inhaled AMK in a Japanese study, despite the use of similar doses of AMK [24]. These differences were presumably because our patients had a high rate of previous usage of injectable aminoglycoside $(58 \%)$ and long periods of previous aminoglycoside use (median, 7.0 months). Generally, CFZ is considered tolerable with no severe adverse effects. In our study, the rate of drug discontinuation due to CFZ side effects was not high, with only a few patients having troublesome adverse effects.

Notably, in our study, treatment for more than 12 months was not significantly associated with favorable outcome in multivariable analysis. This may have been due to the small numbers of patients included in the study. However, our data showed that negative culture conversion was achieved in some patients with treatment for less than 12 months. Further studies are required to confirm this point.

This study has several limitations. First, our data may not be generalizable to other geographic areas and clinical settings. Second, changes in symptoms were assessed using CAT scores, but there is little evidence that it is reasonable to use CAT scores to assess symptom changes in patients with NTM-PD. Third, treatment outcomes were reported based on combination therapy with a relatively short duration. Fourth, a recent study discussed the importance of the CFZ MIC level, but we did not measure the MIC levels of CFZ in this study [36]. Fifth, we included "clinical cure" in the definition of "favorable outcome" because patients who achieved "clinical cure" showed clinical and radiological improvement, and sputum samples could not be obtained in most cases with "clinical cure" due to decreased volume of sputum. However, it is possible that our data overestimated the efficacy of the treatment regimens. Finally, because 15 patients had NTM strains with intermediate resistance to AMK (MIC $32 \mu \mathrm{g} / \mathrm{mL}$ ), the efficacy of our regimen containing inhaled AMK and CFZ may seem weak.

In conclusion, this is the first report of patients with refractory MAC-PD who were treated with inhaled AMK- and CFZ-containing regimens in a standard clinical setting. Our data showed that these regimens could provide favorable outcomes for some patients. However, given the adverse effects, a more effective strategy is needed to maintain these therapeutic regimens.

Supplementary Materials: The following are available online at http://www.mdpi.com/2077-0383/9/9/2968/s1, Figure S1: Cumulative (A) culture conversion rate and (B) microbiological cure rate among patients achieving these outcomes, Table S1: Semiquantitative sputum cultures of patients who did not achieve culture conversion on inhaled amikacin- and clofazimine-containing regimens.

Author Contributions: Study conception and design, B.-G.K. and B.W.J.; Data acquisition and analysis, B.-G.K., H.K., O.J.K., and B.W.J.; Experimental work, H.J.H. and N.Y.L.; Data interpretation and manuscript writing, B.-G.K., S.-Y.B., I.S., and B.W.J.; Critical revision and final approval of the manuscript: B.-G.K., H.K., O.J.K., and B.W.J. All authors have read and agreed to the published version of the manuscript.

Funding: This research received no external funding.

Acknowledgments: We would like to express our heartfelt gratitude and respect to Won-Jung Koh for giving us invaluable guidance and unfailing support from the very beginning of this research. Won-Jung Koh passed away in August 2019. We dedicate this work to his memory. 
Conflicts of Interest: The authors declare no conflict of interest.

\section{Abbreviations}

NTM: nontuberculous mycobacteria; PD, pulmonary disease; MAC, Mycobacterium avium complex; AMK, amikacin; CFZ, clofazimine; MIC, minimum inhibitory concentration; CAT, chronic obstructive pulmonary disease assessment test; $\mathrm{CT}$, computed tomography; FC, fibrocavitary; NB, nodular bronchiectatic; AFB, acid-fast bacillus; MGIT, mycobacterial growth indicator tubes; IQR, interquartile range; ESR, erythrocyte sedimentation rate; FEV ${ }_{1}$, forced expiratory volume in $1 \mathrm{~s}$.

\section{References}

1. Prevots, D.R.; Marras, T.K. Epidemiology of human pulmonary infection with nontuberculous mycobacteria: A review. Clin. Chest Med. 2015, 36, 13-34.

2. Lee, H.; Myung, W.; Koh, W.J.; Moon, S.M.; Jhun, B.W. Epidemiology of nontuberculous mycobacterial infection, South Korea, 2007-2016. Emerg. Infect. Dis. 2019, 25, 569-572.

3. Prevots, D.R.; Shaw, P.A.; Strickland, D.; Jackson, L.A.; Raebel, M.A.; Blosky, M.A.; Montes de Oca, R.; Shea, Y.R.; Seitz, A.E.; Holland, S.M.; et al. Nontuberculous mycobacterial lung disease prevalence at four integrated health care delivery systems. Am. J. Respir. Crit. Care Med. 2010, 182, 970-976.

4. Griffith, D.E.; Aksamit, T.; Brown-Elliott, B.A.; Catanzaro, A.; Daley, C.; Gordin, F.; Holland, S.M.; Horsburgh, R.; Huitt, G.; Iademarco, M.F.; et al. An official ATS/IDSA statement: Diagnosis, treatment, and prevention of nontuberculous mycobacterial diseases. Am. J. Respir. Crit. Care Med. 2007, 175, 367-416.

5. Daley, C.L.; Iaccarino, J.M.; Lange, C.; Cambau, E.; Wallace, R.J.J.; Andrejak, C.; Böttger, E.C.; Brozek, J.; Griffith, D.E.; Guglielmetti, L.; et al. Treatment of nontuberculous mycobacterial pulmonary disease: An official ATS/ERS/ESCMID/IDSA clinical practice guideline. Eur. Respir. J. 2020, 56, 2000535.

6. Xu, H.B.; Jiang, R.H.; Li, L. Treatment outcomes for Mycobacterium avium complex: A systematic review and meta-analysis. Eur. J. Clin. Microbiol. Infect. Dis. 2014, 33, 347-358.

7. Kwak, N.; Park, J.; Kim, E.; Lee, C.H.; Han, S.K.; Yim, J.J. Treatment outcomes of Mycobacterium avium complex lung disease: A systematic review and meta-analysis. Clin. Infect. Dis. 2017, 65, 1077-1084.

8. Pasipanodya, J.G.; Ogbonna, D.; Deshpande, D.; Srivastava, S.; Gumbo, T. Meta-analyses and the evidence base for microbial outcomes in the treatment of pulmonary Mycobacterium avium-intracellulare complex disease. J. Antimicrob. Chemother. 2017, 72 (Suppl. 2), i3-i19.

9. Diel, R.; Nienhaus, A.; Ringshausen, F.C.; Richter, E.; Welte, T.; Rabe, K.F.; Loddenkemper, R. Microbiologic outcome of interventions against Mycobacterium avium complex pulmonary disease: A systematic review. Chest 2018, 153, 888-921.

10. Haworth, C.S.; Banks, J.; Capstick, T.; Fisher, A.J.; Gorsuch, T.; Laurenson, I.F.; Leitch, A.; Loebinger, M.R.; Milburn, H.J.; Nightingale, M.; et al. British thoracic society guidelines for the management of non-tuberculous mycobacterial pulmonary disease (NTM-PD). Thorax 2017, 72 (Suppl 2), ii1-ii64.

11. Floto, R.A.; Olivier, K.N.; Saiman, L.; Daley, C.L.; Herrmann, J.L.; Nick, J.A.; Noone, P.G.; Bilton, D.; Corris, P.; Gibson, R.L.; et al. US cystic fibrosis foundation and european cystic fibrosis society consensus recommendations for the management of non-tuberculous mycobacteria in individuals with cystic fibrosis. Thorax 2016, 71 (Suppl 1), i1-i22.

12. Jhun, B.W.; Yang, B.; Moon, S.M.; Lee, H.; Park, H.Y.; Jeon, K.; Kwon, O.J.; Ahn, J.; Moon, I.J.; Shin, S.J.; et al. Amikacin inhalation as salvage therapy for refractory nontuberculous mycobacterial lung disease. Antimicrob. Agents Chemother. 2018, 62, e00011-18.

13. Martiniano, S.L.; Wagner, B.D.; Levin, A.; Nick, J.A.; Sagel, S.D.; Daley, C.L. Safety and effectiveness of clofazimine for primary and refractory nontuberculous mycobacterial Infection. Chest 2017, 152, 800-809.

14. Griffith, D.E.; Eagle, G.; Thomson, R.; Aksamit, T.R.; Hasegawa, N.; Morimoto, K.; Addrizzo-Harris, D.J.; O'Donnell, A.E.; Marras, T.K.; Flume, P.A.; et al. Amikacin liposome inhalation suspension for treatment-refractory lung disease caused by Mycobacterium avium Complex (CONVERT). A prospective, open-label, randomized study. Am. J. Respir. Crit. Care Med. 2018, 198, 1559-1569.

15. Van Ingen, J.; Totten, S.E.; Helstrom, N.K.; Heifets, L.B.; Boeree, M.J.; Daley, C.L. In vitro synergy between clofazimine and amikacin in treatment of nontuberculous mycobacterial disease. Antimicrob. Agents Chemother. 2012, 56, 6324-6327. 
16. Lee, H.; Sohn, Y.M.; Ko, J.Y.; Lee, S.Y.; Jhun, B.W.; Park, H.Y.; Jeon, K.; Kim, D.H.; Kim, S.Y.; Choi, J.E.; et al. Once-daily dosing of amikacin for treatment of Mycobacterium abscessus lung disease. Int. J. Tuberc. Lung Dis. 2017, 21, 818-824.

17. Kon, S.S.; Canavan, J.L.; Jones, S.E.; Nolan, C.M.; Clark, A.L.; Dickson, M.J.; Haselden, B.M.; Polkey, M.I.; Man, W.D. Minimum clinically important difference for the COPD Assessment Test: A prospective analysis. Lancet Respir. Med. 2014, 2, 195-203.

18. Koh, W.J.; Moon, S.M.; Kim, S.Y.; Woo, M.A.; Kim, S.; Jhun, B.W.; Park, H.Y.; Jeon, K.; Huh, H.J.; Ki, C.S.; et al. Outcomes of Mycobacterium avium complex lung disease based on clinical phenotype. Eur. Respir. J. 2017, 50, 1602503.

19. American Thoracic Society. Diagnostic standards and classification of tuberculosis in adults and children. Am. J. Respir. Crit. Care Med. 2000, 161, 1376-1395.

20. Griffith, D.E.; Adjemian, J.; Brown-Elliott, B.A.; Philley, J.V.; Prevots, D.R.; Gaston, C.; Olivier, K.N.; Wallace, R.J., Jr. Semiquantitative culture analysis during therapy for Mycobacterium avium complex lung disease. Am. J. Respir. Crit. Care Med. 2015, 192, 754-760.

21. Yang, B.; Jhun, B.W.; Moon, S.M.; Lee, H.; Park, H.Y.; Jeon, K.; Kim, D.H.; Kim, S.Y.; Shin, S.J.; Daley, C.L.; et al. Clofazimine-containing regimen for the treatment of Mycobacterium abscessus lung disease. Antimicrob. Agents Chemother. 2017, 61, e02052-16.

22. Woods, G.L.; Brown-Elliott, B.A.; Conville, P.S.; Desmond, E.P.; Hall, G.S.; Lin, G.; Pfyffer, G.E.; Ridderhof, J.C.; Siddiqi, S.H.; Wallace, R.J.J.; et al. CLSI Standards: Guidelines for health care excellence. In Susceptibility Testing of Mycobacteria, Nocardiae, and Other Aerobic Actinomycetes; Clinical and Laboratory Standards Institute: Wayne, PA, USA, 2011.

23. Van Ingen, J.; Aksamit, T.; Andrejak, C.; Bottger, E.C.; Cambau, E.; Daley, C.L.; Griffith, D.E.; Guglielmetti, L.; Holland, S.M.; Huitt, G.A.; et al. Treatment outcome definitions in nontuberculous mycobacterial pulmonary disease: An NTM-NET consensus statement. Eur. Respir. J. 2018, 51, 1800170.

24. Yagi, K.; Ishii, M.; Namkoong, H.; Asami, T.; Iketani, O.; Asakura, T.; Suzuki, S.; Sugiura, H.; Yamada, Y.; Nishimura, T.; et al. The efficacy, safety, and feasibility of inhaled amikacin for the treatment of difficult-to-treat non-tuberculous mycobacterial lung diseases. BMC Infect. Dis. 2017, 17, 558.

25. Jhun, B.W.; Kim, S.Y.; Moon, S.M.; Jeon, K.; Kwon, O.J.; Huh, H.J.; Ki, C.S.; Lee, N.Y.; Shin, S.J.; Daley, C.L.; et al. Development of macrolide resistance and reinfection in refractory Mycobacterium avium complex lung disease. Am. J. Respir. Crit. Care Med. 2018, 198, 1322-1330.

26. Griffith, D.E.; Brown-Elliott, B.A.; Langsjoen, B.; Zhang, Y.; Pan, X.; Girard, W.; Nelson, K.; Caccitolo, J.; Alvarez, J.; Shepherd, S.; et al. Clinical and molecular analysis of macrolide resistance in Mycobacterium avium complex lung disease. Am. J. Respir. Crit. Care Med. 2006, 174, 928-934.

27. Kadota, T.; Matsui, H.; Hirose, T.; Suzuki, J.; Saito, M.; Akaba, T.; Kobayashi, K.; Akashi, S.; Kawashima, M.; Tamura, A.; et al. Analysis of drug treatment outcome in clarithromycin-resistant Mycobacterium avium complex lung disease. BMC Infect. Dis. 2016, 16, 31.

28. Moon, S.M.; Park, H.Y.; Kim, S.Y.; Jhun, B.W.; Lee, H.; Jeon, K.; Kim, D.H.; Huh, H.J.; Ki, C.S.; Lee, N.Y.; et al. Clinical characteristics, treatment outcomes, and resistance mutations associated with macrolide-resistant Mycobacterium avium complex lung disease. Antimicrob. Agents Chemother. 2016, 60, 6758-6765.

29. Morimoto, K.; Namkoong, H.; Hasegawa, N.; Nakagawa, T.; Morino, E.; Shiraishi, Y.; Ogawa, K.; Izumi, K.; Takasaki, J.; Yoshiyama, T.; et al. Macrolide-resistant Mycobacterium avium complex lung disease: Analysis of 102 consecutive cases. Ann. Am. Thorac. Soc. 2016, 13, 1904-1911.

30. Cho, E.H.; Huh, H.J.; Song, D.J.; Moon, S.M.; Lee, S.H.; Shin, S.Y.; Kim, C.K.; Ki, C.S.; Koh, W.J.; Lee, N.Y. Differences in drug susceptibility pattern between Mycobacterium avium and Mycobacterium intracellulare isolated in respiratory specimens. J. Infect. Chemother. 2018, 24, 315-318.

31. Kim, O.H.; Kwon, B.S.; Han, M.; Koh, Y.; Kim, W.S.; Song, J.W.; Oh, Y.M.; Lee, S.D.; Lee, S.W.; Lee, J.S.; et al. Association between duration of aminoglycoside treatment and outcome of cavitary Mycobacterium avium complex lung disease. Clin. Infect. Dis. 2019, 68, 1870-1876.

32. Davis, K.K.; Kao, P.N.; Jacobs, S.S.; Ruoss, S.J. Aerosolized amikacin for treatment of pulmonary Mycobacterium avium infections: An observational case series. BMC Pulm. Med. 2007, 7, 2.

33. Olivier, K.N.; Shaw, P.A.; Glaser, T.S.; Bhattacharyya, D.; Fleshner, M.; Brewer, C.C.; Zalewski, C.K.; Folio, L.R.; Siegelman, J.R.; Shallom, S.; et al. Inhaled amikacin for treatment of refractory pulmonary nontuberculous mycobacterial disease. Ann. Am. Thorac. Soc. 2014, 11, 30-35. 
34. Jarand, J.; Davis, J.P.; Cowie, R.L.; Field, S.K.; Fisher, D.A. Long-term follow-up of Mycobacterium avium complex lung disease in patients treated with regimens including clofazimine and/or rifampin. Chest 2016, 149, 1285-1293.

35. Field, S.K.; Cowie, R.L. Treatment of Mycobacterium avium-intracellulare complex lung disease with a macrolide, ethambutol, and clofazimine. Chest 2003, 124, 1482-1486.

36. Kwak, N.; Whang, J.; Yang, J.S.; Kim, T.S.; Kim, S.A.; Yim, J.J. Minimal Inhibitory concentration of clofazimine among clinical isolates of nontuberculous mycobacteria and its impact on treatment outcome. Chest 2020. [CrossRef]

(C) 2020 by the authors. Licensee MDPI, Basel, Switzerland. This article is an open access article distributed under the terms and conditions of the Creative Commons Attribution (CC BY) license (http://creativecommons.org/licenses/by/4.0/). 